\title{
Research on FBG-Based CFRP Structural Damage Identification Using BP Neural Network
}

\author{
Xiangyi GENG ${ }^{1}$, Shizeng $\mathrm{LU}^{2}$, Mingshun JIANG ${ }^{1}$, Qingmei SUI ${ }^{*}$, Shanshan $\mathrm{LV}^{1}$, \\ Hang XIAO ${ }^{1}$, Yuxi JIA ${ }^{3}$, and Lei JIA ${ }^{1}$ \\ ${ }^{1}$ School of Control Science and Engineering, Shandong University, Jinan, 250061, China \\ ${ }^{2}$ School of Electrical Engineering, University of Jinan, Jinan, 250022, China \\ ${ }^{3}$ Key Laboratory for Liquid-Solid Structural Evolution \& Processing of Materials (Ministry of Education), Shandong \\ University, Jinan, 250061, China \\ *Corresponding author: Qingmei SUI_Ｅ-mail: sdusuiqingmei@163.com
}

\begin{abstract}
A damage identification system of carbon fiber reinforced plastics (CFRP) structures is investigated using fiber Bragg grating (FBG) sensors and back propagation (BP) neural network. FBG sensors are applied to construct the sensing network to detect the structural dynamic response signals generated by active actuation. The damage identification model is built based on the BP neural network. The dynamic signal characteristics extracted by the Fourier transform are the inputs, and the damage states are the outputs of the model. Besides, damages are simulated by placing lumped masses with different weights instead of inducing real damages, which is confirmed to be feasible by finite element analysis (FEA). At last, the damage identification system is verified on a CFRP plate with $300 \mathrm{~mm} \times 300 \mathrm{~mm}$ experimental area, with the accurate identification of varied damage states. The system provides a practical way for CFRP structural damage identification.
\end{abstract}

Keywords: Carbon fiber reinforced polymer; damage identification; FBG sensors; neural network; finite element analysis

Citation: Xiangyi GENG, Shizeng LU, Mingshun JIANG, Qingmei SUI, Shanshan LV, Hang XIAO, et al., "Research on FBG-Based CFRP Structural Damage Identification Using BP Neural Network," Photonic Sensors, 2018, 8(2): 168-175.

\section{Introduction}

Carbon fiber reinforced polymer (CFRP) has shown great potential in the fields of aeronautics, automotive and civil engineering for its outstanding performances [1]. However, CFRP materials are very susceptible to the invisible structural damage, such as delamination or matrix cracking, causing a severe decrease in mechanical properties and threatening the structural safety. Therefore, the structural damage identification of CFRP materials is an important guarantee for the safety in service.
It has been theoretically and practically proved that damages in a structure will cause the variation of dynamic performances of the whole structure. As a global damage identification method, vibration-based damage identification is widely used $[2,3]$. Structures will be excited by external energy, and then structural dynamic responses containing damage information can be monitored by reliable sensors. In recent years, fiber Bragg grating (FBG) has become the ideal choice as sensing element to realize the damage identification of composite

Received: 31 August 2017 / Revised: 7 January 2018

(C) The Author(s) 2018. This article is published with open access at Springerlink.com

DOI: $10.1007 / \mathrm{s} 13320-018-0466-0$

Article type: Regular 
structures for its advantages of precision, anti-interference and ease to establish sensing network [4-6]. Okabe and Yashiro [7] experimentally and numerically investigated the detection of static and fatigue damage extension in holed CFRP laminates using embedded FBG sensors. An inverse numerical-experimental method was developed in [8] to identify the damage based on natural frequency changes measured by FBG sensors. Wang et al. [9] measured the vibration signals under health status and six kinds of damage status using FBG sensors, realizing the identification of damage existence and severity.

Afterwards, how to identify structural damage states from measured vibration data becomes the key issue. The neural network $[10,11]$ is a potentially powerful algorithm because of the ability to learn from past experiences and memorize the patterns in the form of an associative memory, and the special attention has been drawn to damage recognition and classification. Li et al. [12] presented a thorough investigation into a vibration-based damage identification method utilizing dimensionally reduced residual frequency response function data in combination with neural networks to identify locations and severities of damage in numerical and experimental beam structures. Yam et al. [13] utilized the neural network to establish the mapping relationship between the energy variation of the structural vibration responses and damage status (location and severity), realizing damage detection for polyvinyl chloride (PVC) sandwich plates.

Based on the above, we present a simple and practical method of CFRP structural damage identification. The composite laminate of different damage states is excited by active actuation, and the FBG sensor network is applied to detect the dynamic responses. Eigen frequency changes are extracted by the Fourier transform and set to be the damage characteristics, which do not require pre-analysis of structural model. Then, the back propagation (BP) neural network, whose input is the damage characteristics and output is the damage state, is constructed. Damages in this paper are simulated by placing lumped masses with different weights, instead of inducing real damages, providing an easy and cost saving way for experimental researches.

\section{FBG sensing theory and system setup}

\subsection{FBG sensing theory}

FBG is formed by regular and periodic modulated refractive index along the optical fiber core. When a broadband light is propagating in the FBG, a narrow-band of the incident light reflects back. The wavelength of the reflected light $\lambda_{B}$ depends on the effective refractive index of the fiber core $n_{\text {eff }}$ and the grating period $\Lambda$ as follows [14]:

$$
\lambda_{B}=2 n_{\text {eff }} \Lambda \text {. }
$$

Therefore, the reflected Bragg wavelength is sensitive to both strain and temperature changes. Considering the influence of strain only (experiments are conducted under almost constant temperature), the Bragg wavelength variation can be expressed as

$$
\Delta \lambda_{B} / \lambda_{B}=\left(1-P_{e}\right) \cdot \varepsilon=K_{\varepsilon} \cdot \varepsilon
$$

where $P_{e}$ is the photoelastic coefficient, and $K_{\varepsilon}$ is the strain sensitivity constant. Equation (2) shows the linear relationship between the changes of the FBG wavelength and strain evolutions. Therefore, the wavelength of the FBG pasted on the structural surface will shift because of the structural dynamic strain actuated by active excitation approach, which means that FBG sensors can accurately detect the dynamic response signals containing the structural damage information.

\subsection{Demodulation system}

The high-frequency demodulation system in this paper is composed of an amplified spontaneous emission (ASE) light source without flat processing, splitters, circulators, photoelectric conversion and amplification circuit, a data processing unit, and a 
computer, whose diagram is given in Fig. 1. Combining with the edge filter demodulation principle, the linear segment of the ASE light source is used as the edge filter to realize the high-frequency demodulation of the dynamic response signals (shown in Fig. 2). When the reflection peak of the FBG sensor shifts, the reflection peak intensity changes correspondingly due to the modulation effect of the ASE source hypotenuse filter. This change is converted to a voltage signal by the photoelectric conversion and amplification circuit, and is collected and displayed by the computer after being processed by the data processing unit.

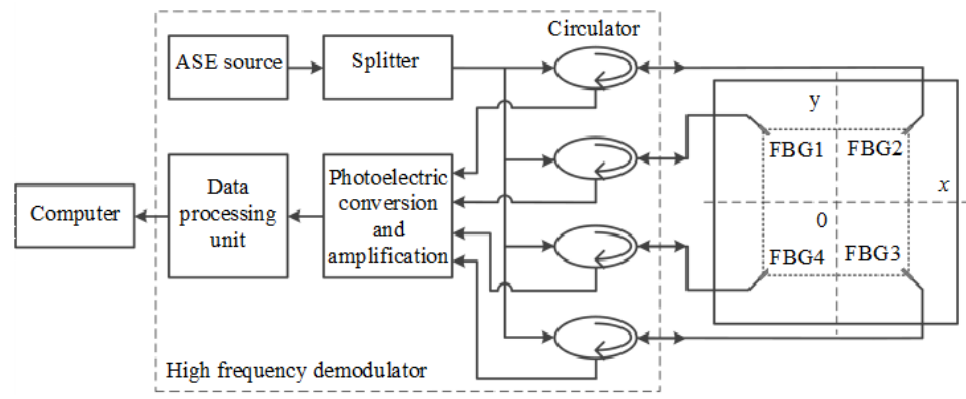

Fig. 1 Diagram of the FBG high frequency demodulation system.

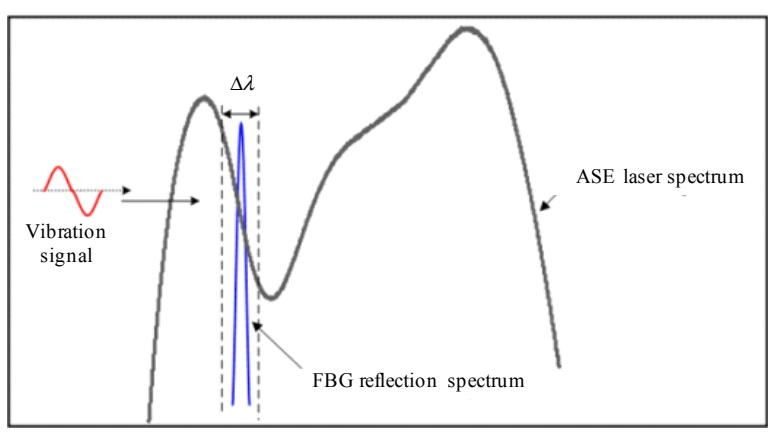

Fig. 2 Principle of edge filter demodulation.

The CFRP plate used in this work is $500 \mathrm{~mm} \times$ $500 \mathrm{~mm} \times 2 \mathrm{~mm}$ with its four edges clamped on the test bench, and a $300 \mathrm{~mm} \times 300 \mathrm{~mm}$ area is selected in the center of the specimen as the experimental area. Four FBG sensors are pasted on the surface of the CFRP plate to construct the sensor networks and detect the dynamic responses. The wavelengths are $1533.27 \mathrm{~nm}, 1533.27 \mathrm{~nm}, 1533.28 \mathrm{~nm}$, and $1533.29 \mathrm{~nm}$. And their locations are $(-150 \mathrm{~mm}$, $150 \mathrm{~mm}),(150 \mathrm{~mm}, 150 \mathrm{~mm}),(150 \mathrm{~mm},-150 \mathrm{~mm})$, and $(-150 \mathrm{~mm},-150 \mathrm{~mm})$, respectively.

\subsection{Damage states and simulation}

Experimentally, there are two ways of constructing the CFRP structural damages, setting actual damages, and inducing simulated damages. Actual damages in composites reduce the stiffness and produce the same result in dynamic responses as mass increase produces (since dynamic response is a ratio of stiffness matrix over mass matrix), i.e. a shift in the eigen frequencies and the corresponding amplitudes [15]. However, the experiments need plenty of composites with the same size and properties but different damage modes, which is costly and hard to achieve. Therefore, the simulation of structural damages appears particularly important. Besides, the damage simulation can eliminate the interference of the CFRP size or properties. So, damages in this work are simulated by adding the known masses, instead of inducing real damage.

In order to verify the feasibility, finite element analysis is conducted. The four edges of the CFRP plate are clamped on the test bench. Structural damages are simulated with lumped masses $(100 \mathrm{~g}$, $200 \mathrm{~g}$, and $500 \mathrm{~g}$ ) placed in the center of the experimental area (presented in Fig. 3). Different weights of the lumped masses simulate different damage degrees. The corresponding damage states are defined as states $1-4$, respectively, as shown in Table 1. The finite element results corresponding to each damage states are given in Fig. 4, revealing that the stress conditions of the composite plate differed obviously after applying different lumped masses. 
With an increase of the weights, the deformation range in the center of the CFRP plate expands. Therefore, the lumped masses on the composite could easily and capably simulate the structural damages.

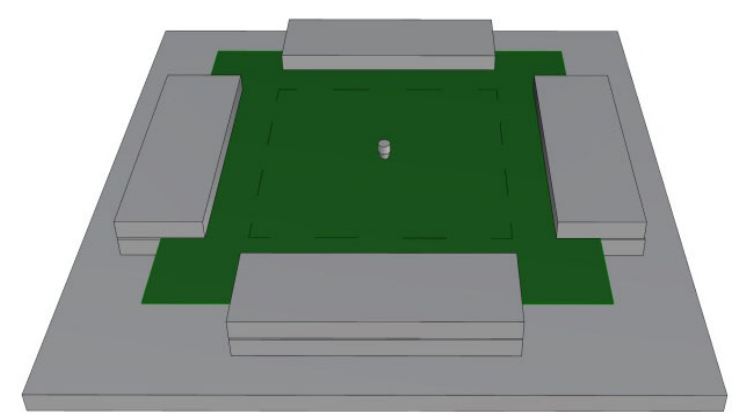

Fig. 3 Damage simulation of clamped CFRP plate.

Table 1 Definition of damage states.

\begin{tabular}{cc}
\hline State & Definition \\
\hline 1 & No damage \\
2 & $100 \mathrm{~g}$ \\
3 & $200 \mathrm{~g}$ \\
4 & $500 \mathrm{~g}$ \\
\hline
\end{tabular}
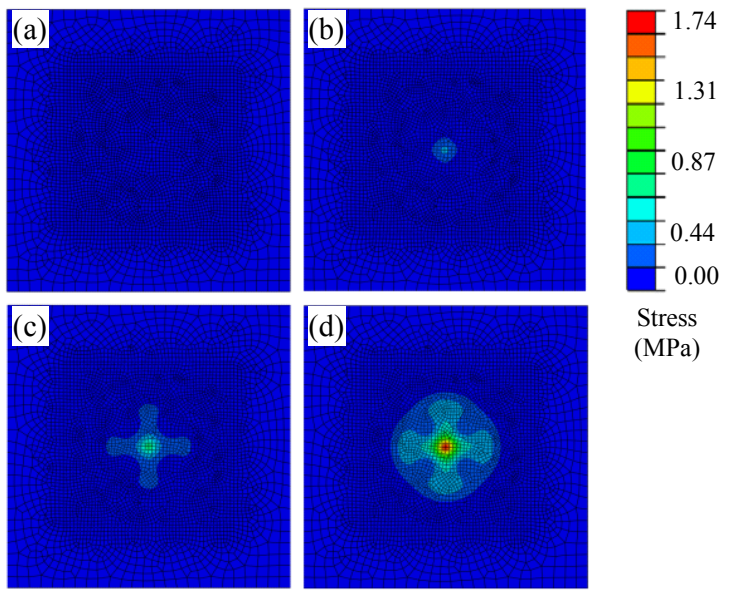

Fig. 4 Stress cloud charts of (a) - (d): damage states $1-4$.

\section{Experiments and analysis}

\subsection{Signal detecting and preprocessing}

The active actuation approach is adopted to identify the structural damage. The excitation is non-destructive and carried out by a $25 \mathrm{~g}$ steel ball impacting the CFRP plate with the form of free fall. The actuation point is located on the $1 / 4$ of the central axis of the experimental area (marked in Fig. 5). The actuation height is set to be $100 \mathrm{~mm}$ to ensure that the excitation energy remained constant during each experiment. Then, the dynamic response signals of different damage states can be detected by the FBG sensor network with the sampling frequency of $500 \mathrm{kHz}$.

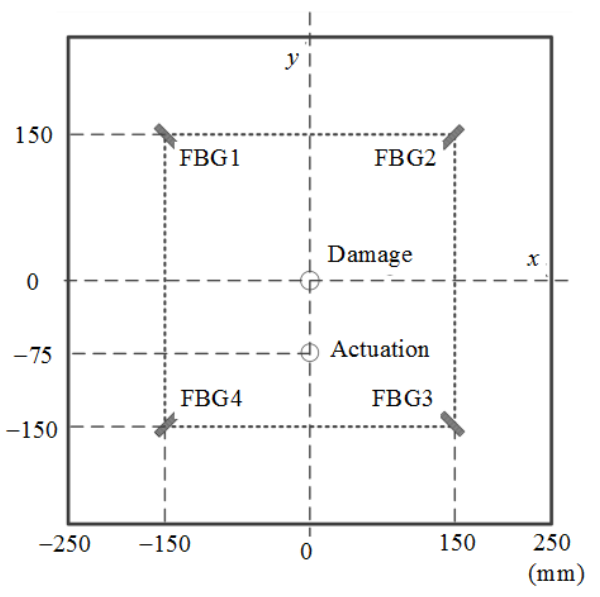

Fig. 5 Schematic of the active actuation experiment.

The prerequisite for the realization of structural damage identification is the accurate extraction of damage characteristics. Taking the signals of different damage states detected by FBG1 as an example [Fig. 6(a)], we can find that there are a large number of high frequency signal components which may be introduced by the light source jitter, internal system noise or environment interference during the experiments. So, the daubechies4 (db4) wavelet is applied in this paper to do the 5-layer discrete wavelet transform denoising before identification. The fifth layer approximation coefficients a5 of the signals are obtained, and the reconstructed signals are given in Fig. 6(b). Then, the amplitude-frequency characteristics are extracted by the Fourier transform, and the results are shown in Fig. 7. It is obvious that the frequency responses vary under different structural damage states. That is to say, there is a certain relationship between the signal amplitude-frequency characteristics and the damage states. Therefore, the frequency responses can be extracted as the damage characteristics. 

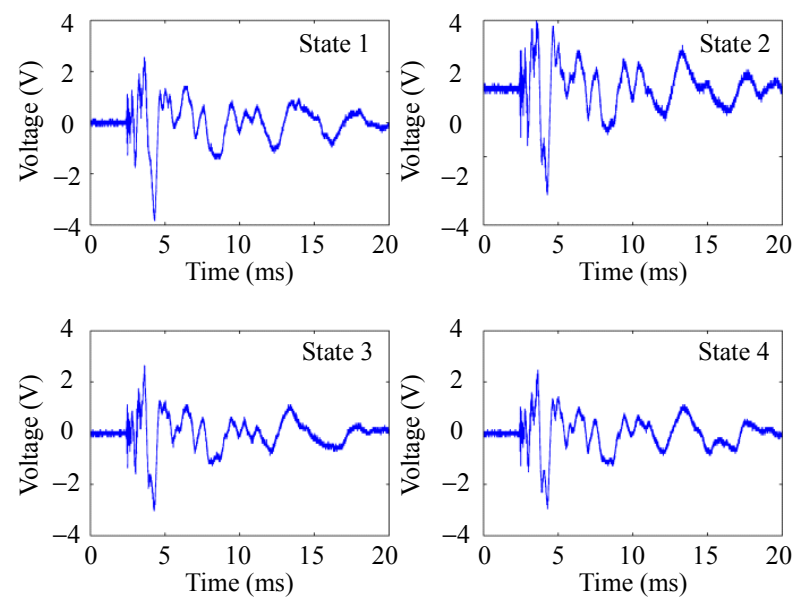

(a)
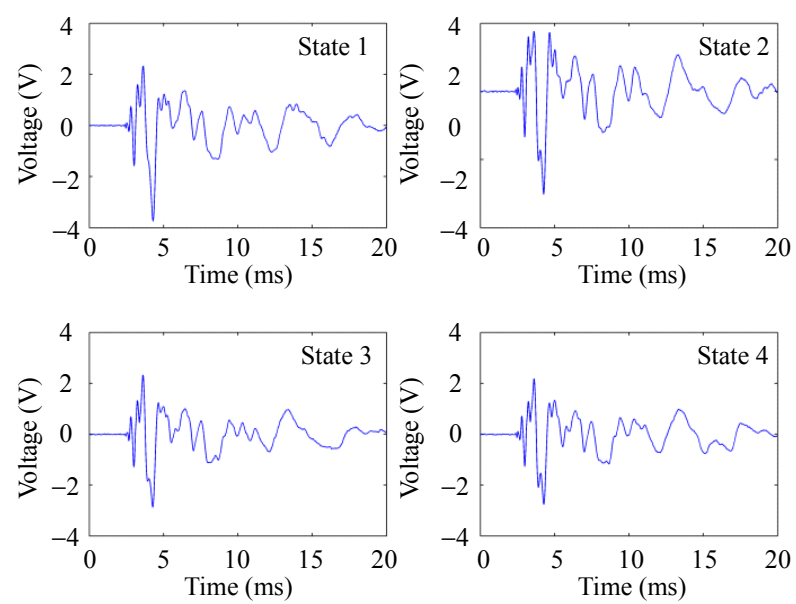

(b)

Fig. 6 Signals detected by FBG1 (a) before and (b) after wavelet transform denoising.

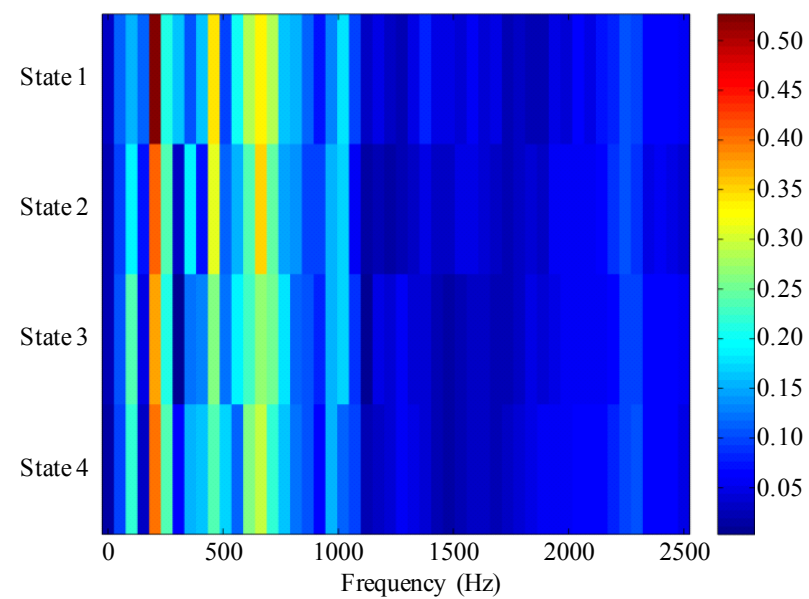

Fig. 7 Amplitude-frequency characteristics of signals detected by FBG1.

\subsection{BP neural network}

The BP neural network is a kind of multilayer feedforward neural network trained by the error back propagation algorithm, which is one of the most widely used neural network models. It can learn and store a large number of input-output model mapping, and need not to describe the mathematical equation of the mapping relationship in advance.

The topology of the BP neural network model includes the input layer, hidden layer, and output layer, as shown in Fig. 8. $x_{j}$ is the input of the $j$ th node of the input layer, where $j=1,2, \cdots, M . w_{i j}$ and $\theta_{i}$ are the weight and threshold of the $i$ th node of the hidden layer to the input layer, respectively, where $i=1,2, \cdots, q . \phi$ is the transfer function of the hidden layer. $\omega_{k i}$ is the weight of the $k$ th node of the output layer to the $i$ th node of the hidden layer, where $k=1,2, \cdots, L . a_{k}$ and $o_{k}$ are the threshold and output of the $k$ th node of the output layer, respectively. In this model, the inputs are the amplitude-frequency characteristics extracted by the Fourier transform, and the output is the damage state (state 1, 2, 3, or 4). Its learning rule is the gradient descent method, which adjusts the weights and thresholds of the network by back propagation, to minimize the squares sum of the network error.

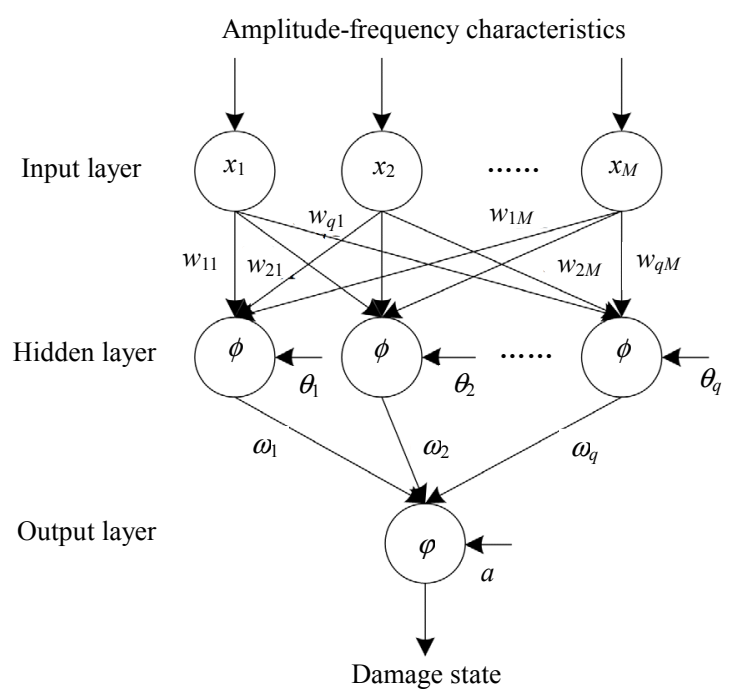

Fig. 8 Topology of the BP neural network.

As shown in Fig. 8, the input of the hidden layer $u_{i}$ is as follows:

$$
u_{i}=\sum_{i=1}^{M} w_{i j} x_{j}+\theta_{i} .
$$

According to the transfer function of the hidden 
layer, the output of the $i$ th node of the hidden layer can be expressed as follows:

$$
y_{i}=\phi\left(u_{i}\right)=\phi\left(\sum_{i=1}^{M} w_{i j} x_{j}+\theta_{i}\right) .
$$

Therefore, the input of the output layer $v$ is as follows:

$$
v=\sum_{i=1}^{q} \omega_{i} y_{i}+a=\sum_{i=1}^{q} \omega_{i} \phi\left(\sum_{i=1}^{M} w_{i j} x_{j}+\theta_{i}\right)+a .
$$

According to (3) - (5), the output of the output layer $o$ is as follows:

$$
\begin{aligned}
o & =\varphi(v)=\varphi\left(\sum_{i=1}^{q} \omega_{i} y_{i}+a\right) \\
& =\varphi\left(\sum_{i=1}^{q} \omega_{i} \phi\left(\sum_{i=1}^{M} w_{i j} x_{j}+\theta_{i}\right)+a\right)
\end{aligned}
$$

Assuming that the excepted output of the output layer is $T$, thus the output error $e$ is as follows:

$$
e=T-o \text {. }
$$

The training of neural networks is completed until the weights and thresholds are constantly adjusted until the desired value is reached.

\subsection{BP neural network training}

Under each damage state, 10 experiments are carried out, respectively, and 40 groups of dynamic response signals are obtained by FBG sensors as training samples of the BP neural network. The sampling frequency is $500 \mathrm{kHz}$ with 10000 sampling points. After denoising, the amplitude-frequency characteristics are extracted by the Fourier transform as the input of the BP neural network. After the Fourier transform, we find that the energy of the frequency response is mainly concentrated on the low frequency part. Accordingly, the first 150 frequency points $(0 \mathrm{kHz}-7.5 \mathrm{kHz})$ is taken as the input of the BP neural network, which can also reduce the dimension of the BP neural network and shorten the computation time. As a result, the number of input layer neurons is $150 \times 4=600$, while the number of output layer neurons is 1 , i.e. the structural damage state.

After the training samples are settled, the number of hidden layer neurons should be determined. An empirical formula is used as follows:

$$
M=\sqrt{n+o}+a .
$$

where $M, n$, and $o$ are the number of hidden layer neurons, number of input layer neurons, and number of output layer neurons, respectively, and $a$ is a constant from 1 to 10 . The calculated scope of hidden layer neurons is from 26 to 35 . Meanwhile, Levenberg-Marquardt (LM) algorithm is induced in order to solve the problem of slow convergence of the gradient descent method. When using the LM algorithm for training samples, it is founded that when the number of hidden layer neurons is 26 , the convergence rate is the fastest, so the number of hidden layer neurons is set to be 26. For avoiding the instability of the system, the learning rate is set to be 0.01 , and the maximum error rate is $1 \mathrm{E}-6$. Using the above parameters to train samples, the authors find that the iteration number changes during $5-7$, so set the iteration number to be 10 . Figure 9 shows that the training results of the BP neural network, revealing that the neural network can accurately learn from the 40 groups of training samples.

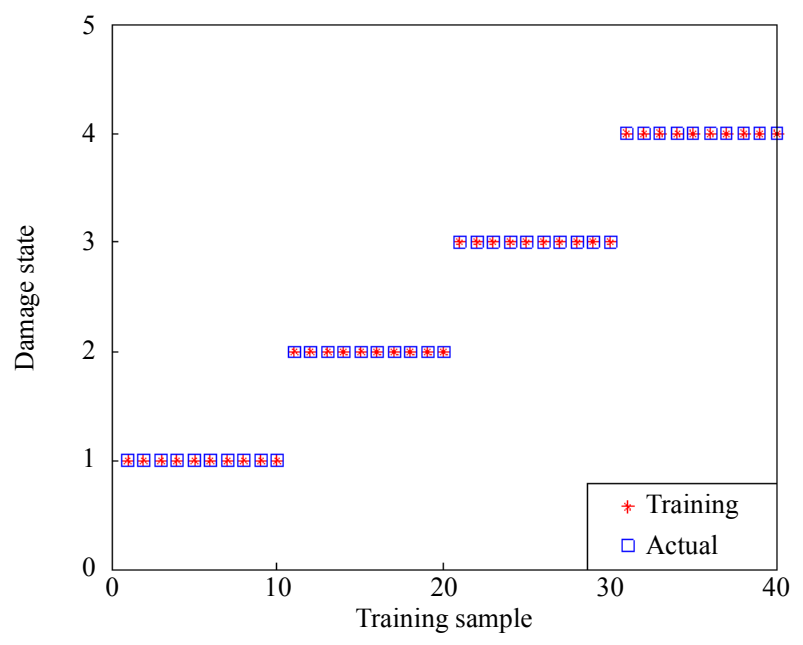

Fig. 9 Training results of the BP neural network.

\subsection{Tests of damage identification}

Another 20 experiments under different damage states are conducted on the CFRP plate, and the dynamic response signals are used as the test samples. The frequency characteristics of the test 
samples are put into the trained network to get the test results, which are presented in Fig. 10. It is obvious that the trained BP neural network can be used to predict the damage states of the test samples accurately.

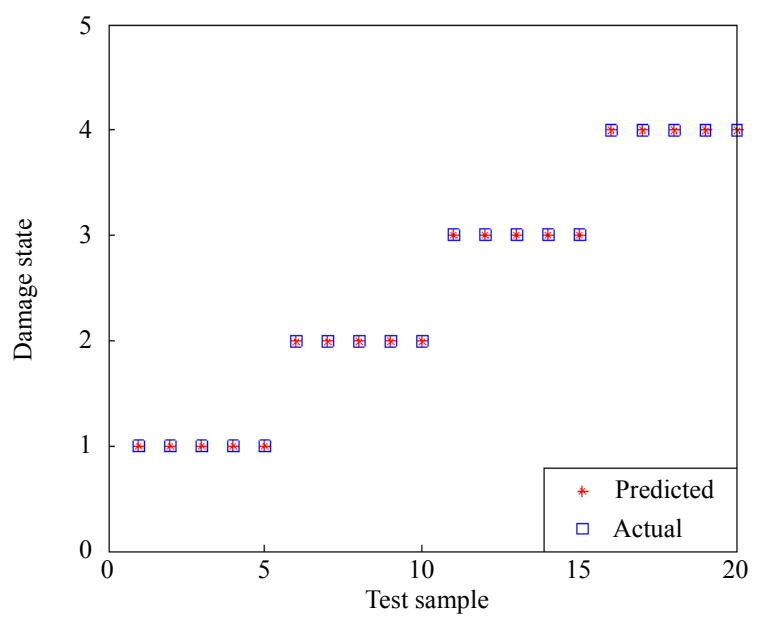

Fig. 10 Predicted results of the BP neural network.

\section{Conclusions}

In this paper, the damage identification method of CFRP structures based on the FBG sensors and BP neural network is investigated and experimentally verified. Dynamic response signals generated by the active actuation approach are captured by a high-frequency demodulation system. Lumped masses with different weights are applied on the CFRP plate to simulate different damage degrees, which are confirmed to be feasible by finite element analysis. The BP neural network, whose input is the amplitude-frequency characteristic and output is the damage state, is designed to identify the damage states. Experiments are carried on a CFRP plate, and the results showed that the damage states are investigated with accurate identification. This paper provides a practical and easy way to realize the CFRP structural damage degree identification.

\section{Acknowledgment}

This work was supported by the National Natural Science Foundation of China under Grant Nos. 41472260 and 51373090, the Natural Science
Foundation of Shandong Province, China under Grant Nos. 2014ZRE27372 and ZR2017BF007, the Fundamental research funds of Shandong University, China under Grant No. 2016JC012, and the Young Scholars Program of Shandong University under Grant No. 2016WLJH30.

Open Access This article is distributed under the terms of the Creative Commons Attribution 4.0 International License (http://creativecommons.org/ licenses/by/4.0/), which permits unrestricted use, distribution, and reproduction in any medium, provided you give appropriate credit to the original author(s) and the source, provide a link to the Creative Commons license, and indicate if changes were made.

\section{References}

[1] W. J. Staszewski, S. Mahzan, and R. Traynor, "Health monitoring of aerospace composite structures-active and passive approach," Composites Science \& Technology, 2009, 69(11-12): 1678-1685.

[2] W. Fan and P. Z. Qiao, "Vibration-based damage identification methods: a review and comparative study," Structural Health Monitoring, 2010, 9(3): 83-111.

[3] H. Y. Hwang and C. Kim, "Damage detection in structures using a few frequency response measurements," Journal of Sound \& Vibration, 2004, 270(1-2): 1-14.

[4] E. Kirkby, R. D. Oliveira, V. Michaud, and J. A. Manson, "Impact localisation with FBG for a self-healing carbon fibre composite structure," Composite Structures, 2011, 94(1): 8-14.

[5] S. R. Di, "Fibre optic sensors for structural health monitoring of aircraft composite structures: recent advances and applications," Sensors, 2014, 15(8): 18666-18713.

[6] P. M. Lam, K. T. Lau, H. Y. Ling, Z. Su, and H. Y. Tam, "Acousto-ultrasonic sensing for delaminated GFRP composites using an embedded FBG sensor," Optics \& Lasers in Engineering, 2009, 47(10): 1049-1055.

[7] T. Okabe and S. Yashiro, "Damage detection in holed composite laminates using an embedded FBG sensor," Composites Part A: Applied Science \& Manufacturing, 2012, 43(3): 388-397.

[8] J. Frieden, J. Cugnoni, J. Botsis, and T. Gmür, "Low energy impact damage monitoring of composites using dynamic strain signals from FBG sensors-part II: damage identification," Composite Structures, 2012, 94(2): 593-600. 
[9] W. Wang, Y. C. Lin, M. R. Zhao, X. Y. Shen, Y. G. Huang, and L. Song, "Damage identification technology based on fiber Bragg grating using SPC and wavelet transform," Journal of Vibration Measurement \& Diagnosis, 2011, 31(5): 566-569.

[10] X. J. Chen, Z. F. Gao, and W. Wang, "Application of BP artificial neural network in structure damage identification," in Proceeding of International Conference on Intelligent Computation Technology and Automation IEEE Computer Society, Changsha, Hunan, China, 2010, pp. 733-737.

[11] P. Selva, O. Cherrier, V. Budinger, F. Lachaud, and J. Morlier, "Smart monitoring of aeronautical composites plates based on electromechanical impedance measurements and artificial neural networks," Engineering Structures, 2013, 56(6): 794-804.

[12] J. C. Li, U. Dackermann, Y. L. Xu, and B. Samali,
"Damage identification in civil engineering structures utilizing PCA-compressed residual frequency response functions and neural network ensembles," Structural Control \& Health Monitoring, 2011, 18(2): 207-226.

[13] L. H. Yam, Y. J. Yan, and J. S. Jiang. "Vibration-based damage detection for composite structures using wavelet transform and neural network identification," Composite Structures, 2003, 60(4): 403-412.

[14] K. O. Hill and G. Meltz, "Fiber Bragg grating technology fundamentals and overview," Journal of Lightwave Technology, 1997, 15(8): 1263-1276.

[15] T. H. Loutas, A. Panopoulou, D. Roulias, and V. Kostopoulos, "Intelligent health monitoring of aerospace composite structures based on dynamic strain measurements," Expert Systems with Applications, 2012, 39(9): 8412-8422. 